\title{
Double fenestration of the ipsilateral internal jugular vein
}

\author{
Mehmet H. Atalar ${ }^{1}$, Gökhan Yillmaz ${ }^{2}$
}

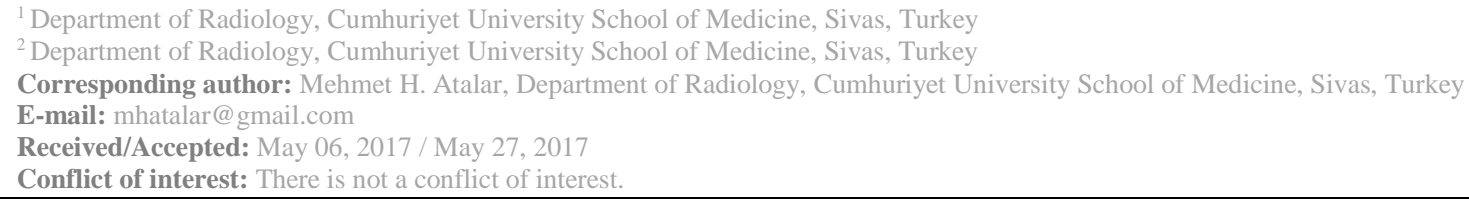

\section{To the editor,}

Internal Juguler vein (IJV) is the largest venous structure in the neck and drains venous blood from cranium, facial region, and the neck. IJV is an important anatomical landmark during surgical and interventional radiological procedures, and a thorough knowledge of its anatomical variations and neighborhood relationships is of paramount significance. Fenestration is a rare malformation of IJV ${ }^{1-3}$.

A 3-year-old female child was referred by an outside center to our center for computed tomography examination for further evaluation of a mass lesion in her neck. Her physical examination was not remarkable for any abnormality. The laboratory studies (including blood cell count, hemoglobin concentration, and hematocrit) were all normal. A contrast-enhanced multislice computed tomography (MSCT) examination was performed using a 16-slice computed tomography device (Brilliance, Philips, Amsterdam, Netherlands). The tomographic sections showed a tortuous and dilated IJV on the right side of the neck; three dimensional (3D) volumetric images were reconstructed using the axial plane images stored on the workstation. A 3D MSCT angiography showed double fenestration in the right IJV, and dilation and tortuosity in its terminal segment (Figures. 1A, B).

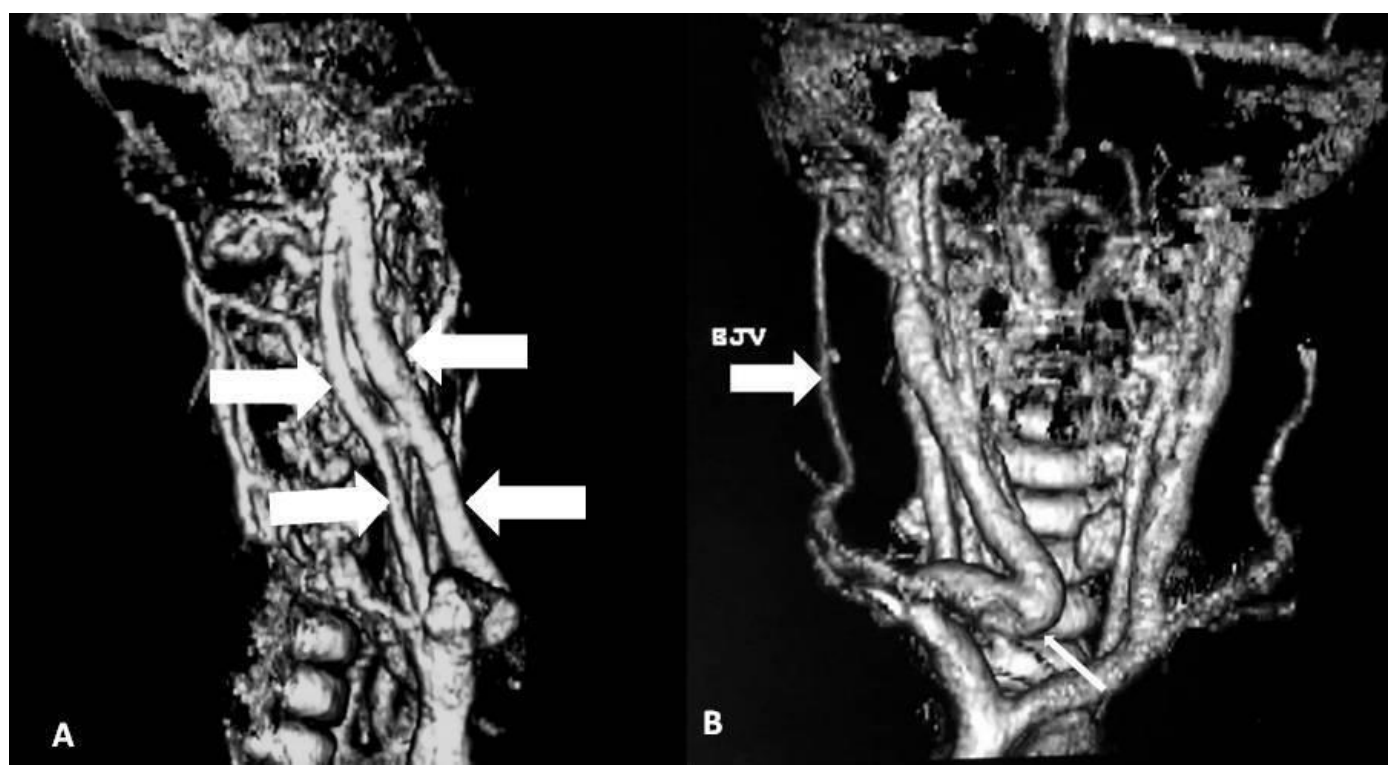

Figure 1. Three dimensional CT volumetric images. (A) A double fenestration is visible in the right IJV on lateral projection (thick white arrows). (B) Antero-posterior projection shows dilation and tortuosity of the right IJV (thin white arrow). The thick white arrow denotes external jugular vein (EJV). 
Additionally, multiple conglomerated lymph nodes were present in bilateral posterior cervical region. The patient was informed about her vascular anomaly and discharged.

Fenestrations and duplications of IJV are rare congenital vascular anomalies of neck. However, there is an important difference between fenestration and duplication. The main difference between these two clinical entities is that in duplication IJV originates as a single vessel from the cranium, branches off into two superiorly, and converges distally with the subclavian vein as two separate vessels, whereas in separation the two veins converge with each other before they converge with the subclavian artery, as in our patient ${ }^{2,4-5}$.

The incidence of duplication is $0.4 \%$ while fenestration has an incidence of less than $1 \%$. These two variations may co-occur with aneurysm and flebectasia. Our patient also had flebectasia and tortuosity in the terminal part of the right IJV. The embryological origin of IJV fenestration is unclear. Vascular, neural, and bony hypotheses have been put forth to explain this variation. Whereas it has been suggested that it occurs at 3-6. Gestational weeks, it has also been reported to occur due to clinical causes such as interventional procedures ${ }^{2-4}$.

IJV fenestrations are usually asymptomatic and usually detected incidentally, as is the case for our patient. However, it is important to show the anatomy of IJV and the accessory nerve during neck dissection in patients with head-neck tumor. The relationship between the accessory nerve and IJV should be clarified in order to protect both structures during neck dissection ${ }^{1,3-5}$.

Modern MSCT technology currently allows the visualization of the supra-aortic arterial and venous structures with a high image quality. The obtained images are reformatted and reconstructed in different planes and used to guide planning of endovascular and surgical treatment ${ }^{3-5}$.

In conclusion, it is of particular importance to have knowledge of this type of anomaly before contemplating surgical and interventional procedures involving the neck region.

\section{REFERENCES}

1. Rossi A, Tortori-Donati P. Internal jugular vein phlebectasia and duplication: case report with magnetic resonance angiography features. Pediatr Radiol. 2001; 31: 134.
2. Nayak BS . Surgically important variations of the jugular veins. Clin Anat. 2006; 19: 544-546.

3. Cvetko E. Unilateral fenestration of the internal jugular vein: a case report. Surg Radiol Anat. 2015; 37: 875-877.

4. Som PM, Shugar JM, Sacher M, Lanzieri $\mathrm{CF}$. Internal jugular vein phlebectasia and duplication: CT features. J Comput Assist Tomogr. 1985; 9: 390-392.

5. Atalar MH, Altuntaş EE, Koşar MI, Uysal I. Multidetector CT findings of an extraordinary fenestration of the internal jugular vein. Diagn Interv Radiol. 2012; 18 : 164-166. 